\title{
Aktuelle systematische Studien zu Tanzbewegungstherapie und psychischer Gesundheit
}

\author{
Iris Bräuninger
}

V orgestellt werden drei Systematische Reviews zur Tanzbewegungstherapie (DMT): Millman und KollegInnen (2020) untersuchen die Auswirkungen von DMT auf die psychische Gesundheit, Aithal und KollegInnen (2021) bewerten die Frage, wie sich DMT bei Kindern mit ASS auf das Wohlbefinden auswirkt, und Kleinlooh und KollegInnen (2021) überprüfen den Effekt von DMT auf KlientInnen mit einer Persönlichkeitsstörung.

\section{Tanzbewegungstherapie und psychische Gesundheit}

In ihrem systematischen Review überprüfen Millman und KollegInnen (2020) die Auswirkungen von DMT auf psychiatrische klinische Populationen. Die Suche erfolgte in den Datenbanken PubMed, Science Direct, World of Science und Clinicaltrials.gov und schloss kontrollierte Studien zu DMT mit verschiedenen psychiatrischen Störungen (u.a. Depression, Schizophrenie, Autismus und somatoforme Störungen) ein. Von den ursprünglich über 3000 Treffern blieben nach Entfernen der Duplikate 594 Studien übrig, wovon 15 den Einschlusskriterien entsprachen: 1 . RCT oder CT, 2. nach 2004 durchgeführt und veröffentlicht, 3. Gruppenintervention, 4. klinisch diagnostizierte TeilnehmerInnen, 5. DMT- oder BPT-Inter- vention (Tanzbewegungstherapie oder Körperpsychotherapie), 6. englischsprachige Studie. Ausschlusskriterien waren körperliche, medizinische oder neurologische Erkrankungen, Reviews oder Metaanalysen.

Die Ergebnisse zeigen einen positiven Effekt der DMT und BPT auf die psychische Gesundheit, v. a. auf Affektive Störungen:

1. Bei Menschen mit Depression

$\rightarrow$ verbessert sich Selbstwertgefühl, Lebensqualität oder globaler Distress nicht signifikant; $\rightarrow$ aber depressive Symptome werden moderat reduziert.

2. Bei Menschen mit Schizophrenie

$\rightarrow$ verbessern sich negative Symptome, psychotische Symptome, psychosoziale Funktion und Fähigkeit, und Wut wird kontrollierbarer; $\rightarrow$ treten diese Effekte jedoch nicht exklusiv in der Interventionsgruppe auf;

3. Bei Menschen mit Autismus

$\rightarrow$ verbessert sich zwar die Empathiefähigkeit nicht,

$\rightarrow$ jedoch reduzieren sich Autismus-Symptome, und das Körperbewusstsein und die Emotionsinferenz, die Selbst-Andere-Wahrnehmung und die Imitationssynchronisation verbessern sich. 4. Bei Menschen mit somatoformer Störung $\rightarrow$ reduzieren sich somatische und depressive Symptome und erhöht sich die subjektive Lebensqualität. 
Die Autorlnnen empfehlen, bei zukünftigen Studien 1) aktive Kontrollgruppen einzuschließen, 2) spezifische DMT-Behandlung bei psychiatrischen Störungen zu untersuchen, 3) die Stichprobengröße zu erhöhen, um die Zuverlässigkeit, Generalisierbarkeit und statistische Power zu verbessern und 4) strengere experimentelle Methoden inkl. Mixed-Method-Designs (quantitative physiologische, neurophysiologische Datenerhebung und subjektive Selbstberichte) anzuwenden und kognitive Neurowissenschaft zu Embodiment und Interozeption einzubeziehen.

\section{Tanzbewegungstherapie und ASS bei Kindern}

Aithal und KollegInnen (2021) bewerteten in ihrem Review anhand der PRIMSA-Kriterien die Wirkung von DMT bei Kindern mit ASS auf das Wohlbefinden. Die Literatursuche wurde in 10 Datenbanken durchgeführt. Einschlusskriterien waren Studien mit 1) Kindern bis max. 16 Jahre, 2. DMT-Intervention, 3. jegliche Arten von empirischen Forschungsstudien, 4. jegliche Bewertungen des Outputs oder des therapeutischen Prozesses. Eingeschlossen wurden neun Studien, welche anhand der Qualitätseinschätzung und des Evidenzgrades von zwei Critical Appraisal Tools für Behandlungskriterien evaluiert wurden (Mixed Methods Appraisal Tool / MMAT und Center for Evidence-Based Medicine ( CEBM). Von ursprünglich 1209 Treffern blieben 859 Studien nach Entfernen der Duplikate übrig, wovon neun den Einschlusskriterien entsprachen. Die erste Forschungsfrage beschäftigte sich mit der Art und Weise, wie DMT bei Kindern mit ASS angewandt wird (therapeutische Rahmenbedingungen, Techniken, Gesamtprozesse, DMT-Intervention, Anzahl / Frequenz der Therapieeinheiten). Die Ergebnisse zeigen, dass DMT potenziell unterschiedliche Aspekte des Wohlbefindens bei Kindern mit ASS fördern kann. Acht aus neun Studien hoben die Verbesserung verschiedener sozialer und kommunikativer Fähigkeiten durch DMT hervor. Die zweite Forschungsfrage beschäftigte sich mit Methoden und Ergebnissen der Datenerhebung: Drei der Studien wiesen ein quantitatives Design auf, vier ein qualitatives und zwei ein Mixed-Methods Design, wovon nur eine einen RCT einschloss.

\section{Tanzbewegungstherapie für KlientIn-} nen mit einer Persönlichkeitsstörung

Der systematische Review mit thematischer Synthese von Kleinlooh und KollegInnen (2021) evaluierte die Wirkung von DMT auf KlientInnen mit einer Persönlichkeitsstörung (PD). Die Suche erfolgte in den Datenbanken Embase, Medline, PubMed, Web of Science, Psyclnfo / Ovid und Scopus. Für die kritische Bewertung der Qualität der Artikel wurde das Critical Appraisal Skills Program für Qualitative Studien verwendet (CASP; 2019), wobei „Level 1“ das höchste Evidenzlevel (Systematische Reviews, RCTs, Fallserien) und „Level 5“ das niedrigste Evidenzlevel (Expertenmeinungen ohne explizite kritische Überprüfung) bewertet (Oxford Centre for Evidence-based-Medicine-Level of Evidence 2009). Von den 570 Treffern blieben nach Entfernen der Duplikate 421 Studien übrig, wovon vier den Einschlusskriterien entsprachen (1. Keine Restriktionen bezüglich Studiendesigns, 2. DMT-Intervention, 3. Peer-reviewed Artikel in Englisch, Deutsch oder Holländisch, 4. Keine Einschränkung bezüglich Setting und Dauer). Alle vier Artikel wurden mit dem niedrigsten Evidenzlevel 5 bewertet. Alle vier Artikel wurden mit einem CASP-Rating von je 3 eingeschätzt, wobei unklar bleibt, ob sich dies auf die Summe der positiven kritischen Bewertungen bezieht (was bedeuten könnte, dass drei aus insgesamt 10 möglichen CASP Ja-Antworten gegeben wurden).

Die thematische Synthese der Ergebnisse ergab sechs übergreifende Themen für DMTInterventionen: 1. Selbstregulation, 2. zwi- 
schenmenschliche Beziehungen, 3. Selbstintegration, 4. Verarbeitung von Erfahrungen, 5. Erkenntnis und Ausdruck sowie 6. Symbolisierung in Bewegung/Tanz. Es konnten keine DMT-Interventionen für PD identifiziert werden. Jedoch wiesen umfangreiche Interventionskomponenten darauf hin, dass in der DMT-Behandlung von PD körperorientierte Strategien eingesetzt und kognitive Strategien für tanzinformierte Aktivitäten zur Selbstregulation integriert werden.

\section{Schlussfolgerung}

Die drei aktuellen systematischen Reviews (SRs) dokumentieren eine erfreuliche Tendenz: Die Forschungsaktivitäten in der DMT sind rege, und die methodische Qualität der SRs sind vielversprechend. Allerdings können die positiven Aktivitäten nicht über den nach wie vor bestehenden dringenden Bedarf an DMT-Primärstudien im Bereich der psychischen Gesundheit hinwegtäuschen. Millman und KollegInnen (2020) fassen die Handlungsfelder deutlich zusammen, wohin sich die DMT-Forschung orientieren sollte: RCTs mit aktiven Kontrollgruppen, Untersuchung spezifischer DMT-Interventionen, Rekrutierung größerer Stichproben und Anwendung experimenteller Methoden inklusive Mixed-Methods-Designs. Hochwertige Forschung in der DMT ist möglich, dies zeigt der Anstieg an Studien mit methodisch hochwertigeren Designs. Sie hat aber ihren Preis, und das DMT-Feld sollte sich der Frage stellen: Wie kann gute DMT-Forschung in Zukunft vermehrt finanziert werden?

\section{Literatur}

Aithal, S., Moula, Z., Karkou, V., Makris, S., Karaminis, T., Powell, J. (2021): A systematic review of the contribution of dance movement psycho- therapy towards the well-being of children with autism spectrum disorders. Frontiers in Psychology 21 (719673), 1-20, https://doi. org/10.3389/fpsyg.2021.719673

Critical Appraisal Skills Programme (2018): CASP Qualitative Checklist. In: casp-uk.net/casptools-checklists, 14.10.2021

Kleinlooh, S. T., Samaritter, R. A., van Rijn, R. M., Kuipers, G., Stubbe, J.H. (2021): Dance movement therapy for clients with a personality disorder: A systematic review and thematic synthesis. Frontiers in Psychology 12, 712, https:// doi.org/doi.10.3389/fpsyg.2021.581578

Millman, L.S. M., Terhune, D. B., Hunter, E. C. M., Orgs, G. (2020): Towards a neurocognitive approach to dance movement therapy for mental health: A systematic review. Clinical Psychology \& Psychotherapy 28, 1-15, https://doi. org/10.1002/cpp.2490

Oxford Centre for Evidence-based-MedicineLevel of Evidence (2009): Homepage. In: www. cebm.net/2009/06/oxford-centre-evidencebasedmedicine-levels-evidence-march-2009, 14.10.2021

Shuper Engelhard, E., Vulcan, M. (2021): The potential benefits of dance movement therapy in improving couple relations of individuals diagnosed with autism spectrum disorder A Review. Frontiers in Psychology 12, 71, https://doi.org/10.3389/fpsyg.2021.619936

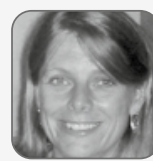

\section{Dr. Iris Bräuninger}

Senior Researcher \& Co-Leiterin Studiengang Psychomotoriktherapie (Hochschule für Heilpädagogik Zürich IVE), Dozentin MA Tanztherapie UAB Barcelona, BTD-Supervisorin / Ausbilderin / Lehrtherapeutin, KMP-Notatorin, Praxis Tanztherapie Supervision Bodensee.

Dr. Iris Bräuninger dancetherapy@mac.com und iris.braeuninger@hfh.ch 